\title{
Introduction: Causation, inferences, and solution types in configurational comparative methods
}

\author{
Tim Haesebrouck ${ }^{1} \cdot$ Eva Thomann $^{2}$
}

Accepted: 12 July 2021 / Published online: 29 July 2021

(C) The Author(s), under exclusive licence to Springer Nature B.V. 2021

\begin{abstract}
This special issue addresses questions of causality and validity of different solution types in configurational comparative methods (CCMs). First, what main parameters characterize the debate about correct causal interpretation of solution types? Second, to what extent has this debate been linked to a theory of causation? The special issue contribution by Mahoney and Acosta bases qualitative comparative analysis (QCA) within a regularity theory of causation integrating type-level inferences and counterfactual cases. Swiatczak clarifies how the different algorithms underlying QCA and Coincidence Analysis (CNA) produce non-identical models. Baumgartner defines and benchmarks QCA solution types against the search target of minimal robust sufficiency. Alamos-Concha et al. identify the conservative solution as most appropriate for a multimethod design combining a counterfactual causal understanding at the cross-case level with an in-depth mechanistic explanation at the within-case level. Finally, Mahoney and Owen develop a general set-theoretic framework for the study of necessity and sufficiency in quantitative research using a counterfactual understanding of causality. Our introduction reviews the state of the art, identifies current limitations and open questions regarding the theoretical basis for causal interpretation of QCA solutions.
\end{abstract}

Keywords Configurational comparative methods · Qualitative comparative analysis $(\mathrm{QCA}) \cdot$ Coincidence analysis $(\mathrm{CNA}) \cdot$ Solution types $\cdot$ Causality

\section{Introduction}

This special issue addresses questions of causality and validity of different solution types in explanatory uses of configurational comparative methods (CCMs). CCMs such as qualitative comparative analysis (QCA) (Ragin 1987/2014) and Coincidence Analysis (CNA) (Baumgartner 2009), have developed into respected methods in the social sciences. The

Tim Haesebrouck

Tim.Haesebrouck@ugent.be

Eva Thomann

Eva.Thomann@uni-konstanz.de

1 Ghent University, Ghent, Belgium

2 University of Konstanz, Konstanz, Germany 
results of both QCA and CNA can be interpreted in terms of sufficiency and necessity and are used for drawing causal inferences. Nevertheless, QCA and CNA use a different analytical approach to produce solutions and suggest different criteria for assessing their validity. While CNA only produces one solution type for each outcome, in the presence of limited empirical diversity, several different solution types can be derived in QCA: conservative, (enhanced) intermediate, and (enhanced) parsimonious solutions (see Oana et al. 2021).

There is an ongoing debate on which one(s) of QCA's different solution type(s) can be causally interpreted. Several publications have argued in favor of one solution. Schneider and Wagemann $(2012,278)$, for example, state that "usually, the enhanced intermediate solution should be at the center of substantive discussion" but also recommend to derive and report all three solution types. Baumgartner (2015), in turn, argues that only the parsimonious solution identifies causally relevant conditions. In contrast, Duşa $(2019,24)$ concludes that the intermediate solution is positioned closest to the true, underlying causal model. Other methodologists and researchers, in turn, suggest that each solution type provides valuable information and researchers should not necessarily only interpret or present one solution (Haesebrouck 2021 (forthcoming); Fiss 2011).

This debate is in progress and reflects several inconclusive aspects of the current state of the art. In particular, it is characterized by the use of different criteria for evaluating the solution types, implicitness about these criteria and the required background assumptions for drawing causal inferences, and/or, more fundamentally, different analytical goals being stated for applying QCA in the first place. Contrary to CNA, there exist different approaches with different analytic goals and understandings of what is a "good" explanation in QCA (Schneider 2018; Thomann and Maggetti 2020). Yet in applied QCA, a variety of solution types are often used inconsistently with these understandings (Thomann and Ege 2020). At the same time, the theoretical basis for establishing criteria for causal interpretation has often remained implicit (Rohlfing and Zuber 2019). Arguably, it would be important for clarifying this basis underlying different QCA approaches, in order to improve both the coherence of the methodology and its explanatory use in empirical research. Absent such clarification, researchers using QCA run the risk of drawing causal inferences without a methodologically sound basis.

Our introduction summarizes the debate on CCM solution types, embeds the special issue contributions therein, and provides structure to it. The five contributions of the special issue, of which Table 1 provides a synopsis, address questions causality and validity of CCM solution types from theoretical and applied perspective. Mahoney and Acosta base QCA within a regularity theory of causation integrating type-level inferences and counterfactual cases. Swiatczak clarifies how the different algorithms underlying QCA and CNA produce non-identical models. Baumgartner defines and benchmarks QCA solution types against the search target of minimal robust sufficiency. Alamos-Concha et al. identify the conservative solution as most appropriate for a multimethod design combining a counterfactual causal understanding at the cross-case level with an in-depth mechanistic explanation at the within-case level. Finally, Mahoney and Owen develop a general set-theoretic framework for the study of necessity and sufficiency in quantitative research using a counterfactual understanding of causality.

We now review the state of the art and summarize the special issue contributions to address two questions. First, what are the main parameters characterizing different positions in the debate about solution types? After discussing the main differences between QCA and CNA, we summarize the debate on QCA solution types based on the distinction between different approaches to QCA. Second, to what extent have different views on correct causal interpretation of CCM results been linked to a theoretical view about causation? 


\begin{tabular}{|c|c|c|}
\hline 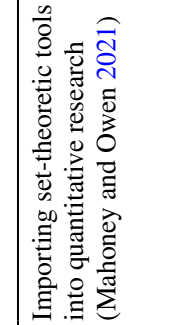 & 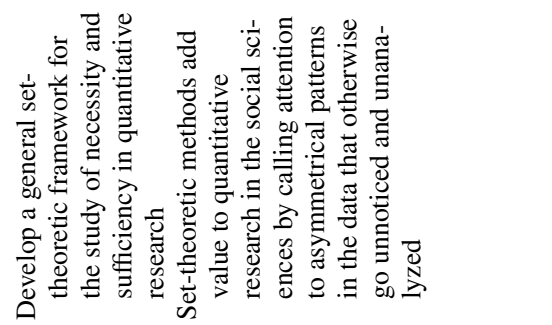 & 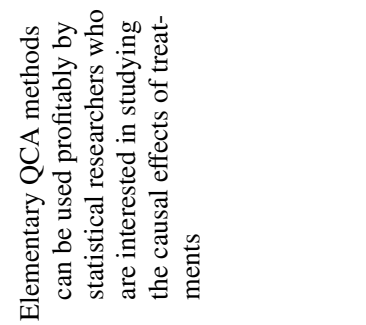 \\
\hline 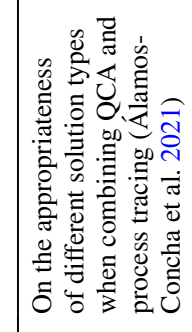 & 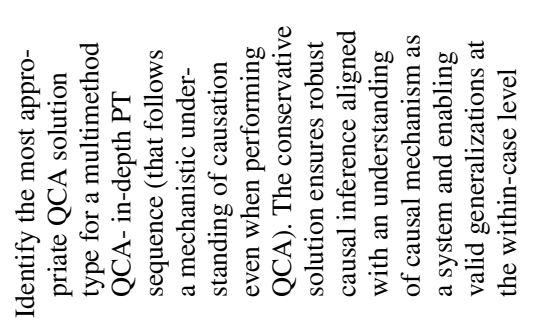 & 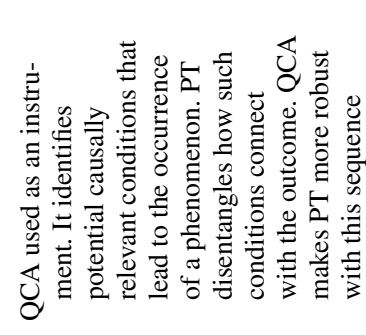 \\
\hline 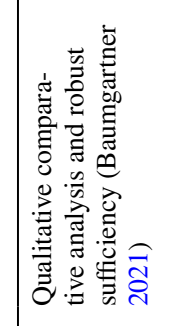 & 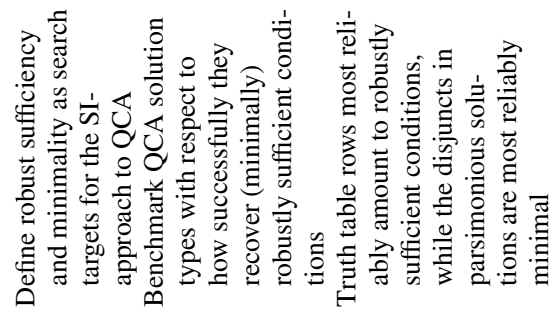 & 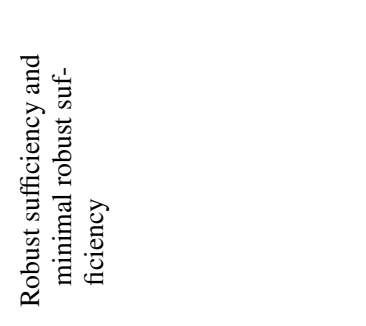 \\
\hline 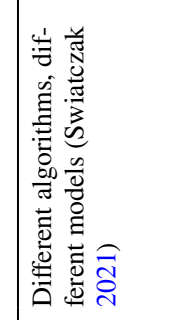 & 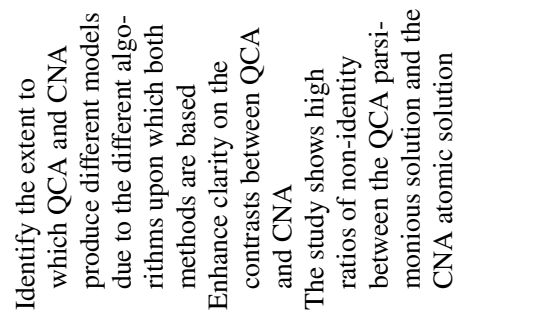 & 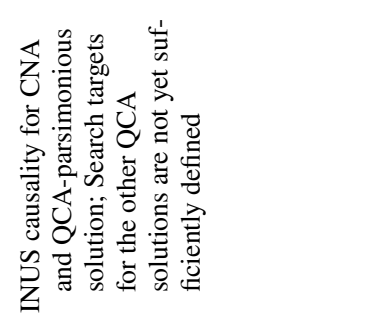 \\
\hline 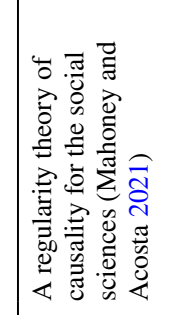 & 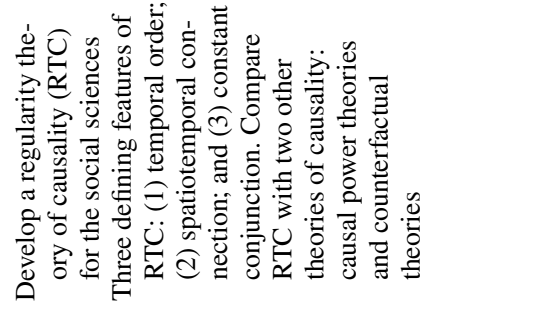 & 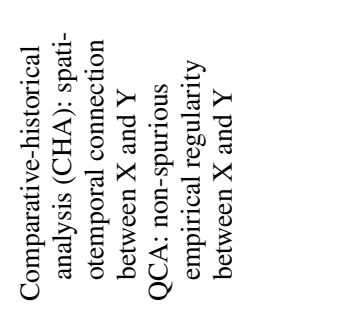 \\
\hline 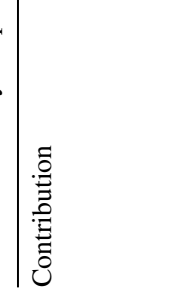 & 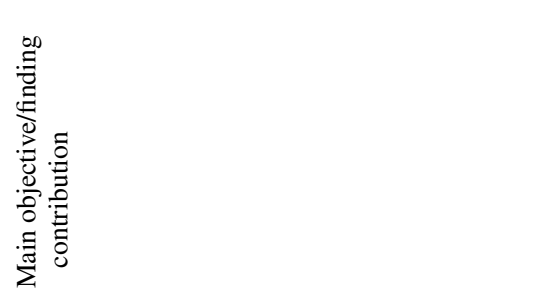 & 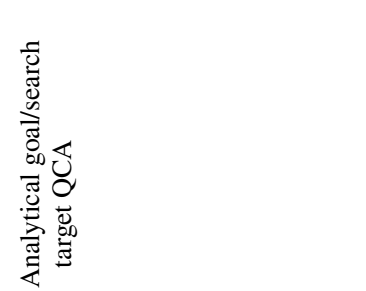 \\
\hline
\end{tabular}




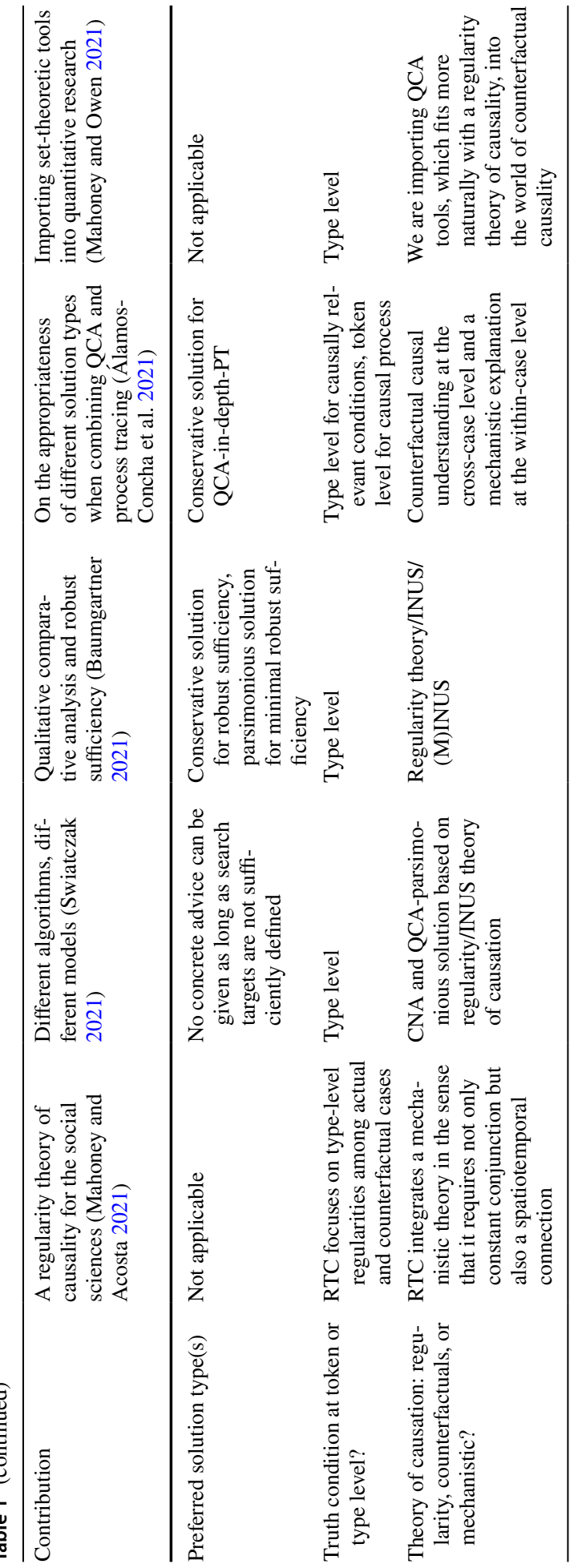


We discuss four theories of causation: regularity theories, token- and type-level counterfactual theories and mechanistic theories. Based on this, we identify open questions and limitations for providing QCA with a theoretical basis for causal interpretation of solution types.

\section{Embedding the debate on CCMs: QCA and CNA compared}

QCA and CNA are the currently most prominent members of the CCM family, which both result in solutions that can be interpreted in terms of necessity and sufficiency and are used for drawing causal inferences. Nevertheless, these two methods use a different analytical approach to produce solutions and judge their validity. As these differences sometimes go unnoticed or are conflated in scholarly discussions, we clarify them here.

QCA, the older of the two methods, uncovers several aspects of causal complexity, also called multiple conjunctural causation (Ragin 1987/2014). Conjunctural causation implies that effects are often produced by a combination of conditions, multiple causation or equifinality implies that several of such combinations can be sufficient for the same outcome, and causal asymmetry refers to the fact that necessary and sufficient causes generally only work in one direction. To uncover complex causal relations, QCA relies on truth tables, which list all logically possible conjunctions of conditions and assigns the cases to them. Based on the consistency parameter, it is assessed for each row of the truth table whether it is sufficient for the outcome. A top-down search strategy called "logical minimization" is then applied on all those truth table rows that pass the consistency test. Its first step consists of pairwise comparisons of primitive sufficient expressions to eliminate redundant conjuncts, which yields the prime implicants. Second, so-called logically redundant prime implicants are eliminated (Oana et al. 2021).

This results in a sufficient solution consisting of disjunctions of conjunctions of socalled "INUS conditions" (Insufficient but Necessary parts of a condition that is Unnecessary but Sufficient). The coverage of this solution indicates the extent to which the sufficient QCA solution accounts for the occurrence of the outcome. Separately and without resorting to truth tables, QCA also explores the presence of (single or complex) necessary conditions for the outcome (Oana et al. 2021).

As soon as the truth table has rows that are not populated by empirical cases, we observe what is called limited empirical diversity (Oana et al. 2021). For deriving a QCA solution, a decision on how to treat the so-called "logical remainders" (the empirically unobserved truth table rows) is required. This decision entails counterfactual assumptions about logical remainder rows: how to deal with the uncertainty regarding the outcome this configuration would produce, if it were empirically observed? The conservative (or complex) solution assumes that absent evidence, none of these logical remainder rows is sufficient for the outcome, and hence does not include them into logical minimization. The parsimonious solution, on the other hand, includes any logical remainder row into logical minimization by assuming it to be sufficient for the outcome, if including that row leads to the further elimination of redundancies. Finally, intermediate solutions can be derived which pay attention to the substantive content of counterfactuals, based on the existing body of theoretical and empirical knowledge. Striking a balance between complexity and parsimony, intermediate solutions further distinguish between plausible and/or tenable, as opposed to implausible and/or untenable counterfactual assumptions (Ragin 2008; Schneider and Wagemann 
2013). In the next section we discuss the ongoing debate on the validity criteria of QCA's solution types and on which solution(s) can be causally interpreted.

Like QCA, CNA also produces solutions that correspond to disjunctions of sufficient combinations for the outcome that capture conjunctural causation and equifinality. However, unlike QCA, CNA can straightforwardly analyze causal structures with multiple outcomes and does not require specifying which factors are causes and which are effects prior to the analysis (Baumgartner 2009, 2013). In consequence, CNA uncovers more complex causal structures, like causal chains and common cause structures. ${ }^{1}$ In contrast to QCA, CNA applies a bottom-up search strategy that does not actively rely on truth tables or counterfactuals (Baumgartner and Ambühl 2018). It starts by assessing the sufficiency of individual conditions for each outcome. If single conditions do not meet a predetermined consistency threshold, conditions are conjunctively added until this threshold is met. This results in a set of minimal sufficient conditions for each outcome. Subsequently, these sufficient conditions are disjunctively conceited to minimally necessary conditions. This results in an atomic solution for each outcome, which represents a minimally necessary disjunction of minimally sufficient combinations. Lastly, these atomic solutions are combined in "complex models", that correspond to complex causal structures with multiple outcomes.

CNA requires solutions to consist of redundancy-free disjunctions of redundancy-free INUS configurations with high enough solution consistency and coverage to amount to a complex sufficient and necessary condition for the outcome (Baumgartner and Ambühl 2018; Baumgartner 2009). Crucially, in order to be considered causally correct, CNA results have to be completely redundancy-free and the model has to amount to an equivalent (i.e. necessary and sufficient) set of the outcome set (Baumgartner and Ambühl 2018). CNA, thus, produces only one solution type for each outcome even in the presence of limited diversity, which is referred to as the atomic solution formula. The last step of CNA builds a complex solution formula, which encompasses all atomic solution formulas.

Swiatczak (2021) more comprehensively discusses the differences between the analytical protocols of QCA and CNA and examines the impact of these differences on the results of these methods. The simulations she presents show that QCA's parsimonious solution and CNA's atomic solution formula are identical only if there is no noise in the data and consistency and coverage thresholds are fixed at 1 . However, when applied to less than perfect data, these solutions are rarely identical, due to the different algorithms upon which both methods are based. Moreover, she argues that contrary to CNA, search targets are not yet sufficiently defined for QCA solutions.

Table 2 summarizes the differences between CNA and QCA. Compared to QCA, CNA models additional aspects of causal complexity and derive results differently. The question of the appropriate solution type only poses itself in QCA, given that CNA only produces one solution type for each outcome. Therefore we now summarize this debate focusing on QCA.

\footnotetext{
${ }^{1}$ QCA can replicate similar structures, but might require drawing contradictory assumptions on logical remainders (Duşa 2018; Thiem 2015).
} 
Table 2 QCA and CNA compared

\begin{tabular}{lll}
\hline & QCA & CNA \\
\hline Truth table based & Yes (for sufficiency) & No \\
Search strategy & Top-down, truth table based & Bottom-up, based on parameters of fit \\
Deals with limited diversity & Explicitly via counterfactual & No counterfactual assumptions \\
& assumptions & \\
Resulting solution types & Conservative/complex & Atomic solution formula \\
& Intermediate & Complex solution formula \\
& Parsimonious & \\
Aspects of complexity modeled & Conjunctural causation & Conjunctural causation \\
& Equifinality & Equifinality \\
& Asymmetry & Asymmetry \\
& & Causal chain \\
& & Common cause \\
\hline
\end{tabular}

${ }^{\mathrm{a}}$ Although it can be argued that the results of CNA do have implications for logical remainders

\section{Approaches to QCA and the debate surrounding solution types}

Within the field of QCA scholars we observe a diversity of analytic approaches which, while using the same technical algorithm, differ in important aspects (Thomann and Maggetti 2020). Accordingly, the current literature lacks an explicit, unified definition of what is deemed a "correct" QCA result (Baumgartner and Thiem 2020). Following Thomann and Maggetti (2020), we focus here on different approaches to cases and to explanation. We identify parameters that provide the background for these approaches to determine a causally correct QCA result and the preference of certain solution types: the use of withincase evidence, the basis of inference needed for causal interpretation, the causal interpretation of necessity and/or sufficiency, the emphasis on INUS causation, and the treatment of uncertainty when there is limited empirical diversity.

\subsection{Approach to cases: case-oriented vs. condition oriented}

QCA scholars differ concerning their approach to cases, that is, how they integrate inferences about specific cases (Thomann and Maggetti 2020). Case-oriented QCA sees the use of within-case evidence as a paramount definitional element of QCA which is used to understand the complexity of cases and to ensure valid description and explanation (Berg-Schlosser et al. 2009; Ragin 1987/2014; Schneider and Wagemann 2012). Ragin (1987/2014, 187), for example, argued that "to construct a useful truth table, it is necessary to gain familiarity with the relevant theories, the relevant research literature, and, most important of all, the relevant cases." However, a condition-oriented QCA has emerged which does not integrate case studies at all. Instead QCA results are interpreted as representing types of cases and assessed based on parameters of fit (consistency and coverage) and robustness (Greckhamer et al. 2013).

The question to what extent case-orientation is required for the validity of QCA results is debated (Rutten 2020; Rihoux 2013). A case-oriented approach does not base statements of necessity or sufficiency purely on the consistency parameter, but additionally requires a (relative) absence of empirical cases whose qualitative set membership scores contradict these statements (also called contradictory cases or deviant cases consistency in kind) 
(Ragin and Rihoux 2009; Schneider and Wagemann 2012). Moreover, case-oriented QCA scholars use case studies in order to verify whether INUS configurations in QCA solutions translate into causal mechanisms at the within-case level (Rihoux and Lobe 2015; Rohlfing and Schneider 2018). Condition-oriented QCA scholars too contend that "if you wish to establish causation, you need to identify the underlying mechanism(s) at work" (Rubinson et al. 2019), but they allow for doing so by drawing upon substantive and/or theoretical knowledge other than within-case evidence.

To summarize, case-oriented QCA identified necessary and/ or sufficient causal conditions by assessing their consistency including the (relative) absence of deviant cases consistency in kind and by assessing the presence of a causal mechanism at the within-case level. Conversely, condition-oriented QCA does not integrate knowledge about specific cases, but requires a result to have a high consistency score, be robust, and a plausible underlying mechanism be supported by substantive and/ or theoretical knowledge.

\subsection{Approach to explanation: substantive interpretability or redundancy-free models}

A second dimension on which QCA scholars differ concerns their approach to explanation, which regards the question what makes for a valid explanation of a QCA result and its causal interpretability. Thomann and Maggetti (2020) differentiate between an approach that emphasizes substantive interpretability (the "SI-approach") and an approach emphasizes redundancy-free models (the "RF-approach"). Schneider (2018) discusses these approaches as QCA “realists" versus "idealists”. Both approaches refer to Mackie's INUStheory of causation and agree that sufficiency and necessity alone are not sufficient to draw causal inferences, but disagree "on the criteria that render certain necessary and sufficient causally interpretable", that is, what to base causal inferences in QCA on (Thomann and Maggetti 2020).

The SI-approach argues that social scientific inquiry is dependent on "the state of existing theoretical and substantive knowledge in the social scientific community at large" (Ragin and Sonnett 2005, 190). Inferences with QCA should not only be based on the data at hand, but also be supported by theoretical, conceptual, and substantive knowledge. In contrast, the RF-approach draws causal inferences from datasets without incorporating prior theoretical or substantive knowledge in the analysis and keeps assumptions needed for causal analysis to a minimum. This approach argues that QCA identifies causally relevant conditions by removing redundancies from sufficient and necessary conditions. Like CNA, the RF- approach suggests that QCA solutions should represent minimally (or redundancyfree) necessary disjunctions of minimally (or redundancy-free) sufficient combinations.

The SI- and RF-approach to QCA have a different view on which QCA solutions should be produced and/or should be at the center of substantive interpretation. The SI-approach postulates that the three solution types should be produced and are valid, but often advises to focus on the (enhanced) intermediate solution. In contrast, the RF-approach argues that only the parsimonious solution should be produced and causally interpreted. However, the debate is more sophisticated than a one-dimensional distinction between a "pro-intermediate" SI-approach and a "pro-parsimonious" RF-approach would suggest. While the SIapproach explicitly builds on the body of theoretical, conceptual, and substantive knowledge, the RF-approach exclusively draws inferences from the data at hand. Approaches to explanation also differ as regards the causal interpretation of necessity and sufficiency, the interpretation of INUS conditions, and the treatment of limited diversity. 


\subsubsection{Causal interpretation of necessity and sufficiency}

The SI-approach and the RF-approach both interpret the results of QCA in terms of necessary and sufficient (combinations of) conditions, but differ on whether there can be causally interpretable sufficiency without necessity, as well as on the specific criteria for interpreting QCA solutions as causal.

The SI-approach allows for the interpretation of necessary but not sufficient conditions. Accordingly, the analysis of necessity is conducted separately from (and often before) the analysis of sufficiency (Schneider and Wagemann 2012, 278). The SIapproach also allows to causally interpret solutions that are sufficient but not necessary. The SI approach applies three overarching criteria for establishing the presence of a (causal) necessary or sufficient conditions: empirical consistency, empirical importance, and substantive importance (Schneider 2018; Oana et al. 2021). An empirically important sufficient condition covers a large share of the outcome set and is given when the condition set is neither much bigger than the outcome set, nor does it approximate a constant (Oana et al. 2021). The degree to which a sufficient solution is necessary is reflected by its coverage, which is interpreted as a measure of empirical relevance. There is no generally accepted lower-bound threshold for coverage in the SI-approach, although a minimum threshold of 0.5 has recently been suggested (Oana et al. 2021, 278). A low coverage indicates low empirical importance, but does not suggest that the sufficient solution is invalid as it can still be substantively important (Schneider and Wagemann 2012, 148).

The SI-approach emphasizes that QCA solutions should not only be empirically consistent and empirically important sufficient conditions, but should also be "substantively important" or "meaningful" (Oana et al. 2021, 206). As argued by Ragin (2008, 53), "the interpretation of any set-theoretic relation as either necessary or sufficient must be built on a solid foundation of theoretical and substantive knowledge". The question of substantive importance refers to whether a set relation is meaningful in light of external knowledge stemming from theory, concepts, and cases, and what can be learned from the results (Oana et al. 2021, 278). Duşa $(2019,12)$, in turn, introduces the concept of robust sufficiency: a solution is robustly sufficient if it "guarantees the outcome will always occur" (emphasize added). Arguably, identifying such a "robust" sufficient combination would require knowing the full cause of an effect, which seems beyond the grasp of empirical research. Other authors argue that the goal of QCA is arriving at a solution that allows for a clearly limited, "modest" degree of generalization (Thomann 2020:264) "Modest generalization" is defined by Berg-Schlosser, De Meur, Ragin and Rihoux, 2009:12) as the formulation of propositions that "we can apply, with appropriate caution, to other similar cases-e.g. cases that share a reasonable number of characteristics with those that were the subject of the QCA". The SI-approach, thus, maintains that QCA-solutions should be more than only consistently sufficient, but also be "meaningful", "substantively important" and/or "robustly" sufficient.

Baumgartner (2021) argues that there is no exact definition for search target of the SI-approach. Therefore, his contribution proposes exact definitions of two possible search targets for the SI approach: robust sufficiency and minimal robust sufficiency. First, Baumgartner (2021) provides an alternative definition of robust sufficiency, which makes clear in what sense a QCA solution can be expected to "guarantee" an outcome. More specifically, QCA solutions can only guarantee an outcome in an idealized background, in which enablers are constantly instantiated, and relative to the set of analyzed 
factors. Second, Baumgartner (2021) introduces the concept of minimal robust sufficient conditions, which are redundancy free robustly sufficient conditions. In a series of simulation experiments, Baumgartner (2021) assesses the performance of the different QCA solution types in recovering (minimal) robust sufficient conditions. He finds that the truth table rows most reliably amount to robustly sufficient conditions, while the disjuncts in parsimonious solutions are most reliably minimal. Hence, he recommends the use of conservative solution for robust sufficiency, and of the parsimonious solution for minimal robust sufficiency.

The RF-approach, in turn, maintains that solutions can only be causally interpreted if they are minimally necessary and sufficient. Interpreting sufficient combinations that are not part of necessary disjunctions can result in causal fallacies (Thiem and Baumgartner 2016). The RF-approach explicitly states that QCA only guarantees fallacy-free causal inferences if the assumption of configurational homogeneity is adopted (Baumgartner and Thiem 2020). Data is homogeneous if there are no unmeasured causes (causally relevant conditions that were not included in the analysis) that have an impact on the outcome that is not mediated through the included conditions. ${ }^{2}$ If a QCA solution does not correspond to a necessary disjunction, this implies that relevant conditions were not included and configurational homogeneity cannot be assumed. Second, according to the RF-approach, conditions are only causally relevant if they are non-redundant parts of sufficient combinations and this sufficient combination is a non-redundant parts of a necessary disjunction. In consequence, identifying necessary conditions before the analysis of sufficiency does not make sense according to this approach.

\subsubsection{Treatment of limited diversity}

One of the most contentious differences between the two approaches is related to the issue of limited diversity. Limited diversity arises from low case-condition ratios, the clustered nature of social reality, and/or the presence of logically impossible combinations of conditions (Schneider and Wagemann 2012). The two approaches recognize that limited diversity has an impact on the results of QCA and creates uncertainty on which conditions are actually indispensable parts of sufficient combination and, thus, causally relevant. However, the two approaches propose different ways to deal with this uncertainty.

The SI-approach focuses on the content of the configurations represented by logical remainder rows and suggests that researchers need to decide on how to treat logical remainders in the analysis (Schneider and Wagemann 2012, 151-177; Ragin 2008, 147-166; Ragin and Sonnett 2005). Locating itself in a broader tradition of counterfactual reasoning in comparative research, this approach emphasizes the importance of making meaningful, "good" counterfactual assumptions (Ragin 2008). Using substantive and theoretical knowledge to assess the plausibility and tenability of counterfactual claims is crucial for the validity of results of QCA and provides a knowledge-based method for addressing the problem of limited diversity (Ragin 2008:173). The SI-approach uses Standard Analysis (Ragin 2008) or Enhanced Standard Analysis (Schneider and Wagemann 2013) to derive intermediate solutions with justified counterfactual assumptions. If no such assumptions can be made, proponents of the SI approach resort to conservative solutions. However, the

\footnotetext{
${ }^{2}$ Proponents of the SI approach have discussed this issue by referring to omitted variable bias, see Radaelli and Wagemann (2019).
} 
SI-approach often cautions against the use of parsimonious solutions, as some simplifying assumptions may lack plausibility and/or tenability. Hereby, the SI-approach avoids that causally relevant conditions are removed from sufficient combinations. However, deriving conservative and intermediate solutions entails a risk of overly complex results containing causally irrelevant conditions, which should not be interpreted causally.

Conversely, the RF-approach only produces one solution type, which corresponds to the parsimonious solution. Only the parsimonious search strategy arrives at a solution that is guaranteed to eliminate all redundant conditions from sufficient combinations. In consequence, this is the only solution that corresponds to a minimally necessary disjunction of minimally sufficient combinations and, thus, the only solution that successfully identifies the conditions for which the data contains evidence that they are causally relevant (Thiem et al. 2020; Thiem 2019). However, it has been explicitly recognized by proponents of this approach that limited diversity can result in incomplete models: not all of the analyzed conditions that are causally relevant will be included in (each path of) the solution in which they are indispensable (Baumgartner and Ambühl 2018). Thus, the interpretation of the resulting models should recognize the potential incompleteness of the configurations.

Table 3 summarizes the differences between the SI and the RF approach to QCA. The SI- and RF-approach, thus, use different bases to draw causal inferences. The SI-approach suggests that an analysis needs to draw on existing theoretical and substantive knowledge to make simplifying assumptions and for interpreting results. It allows for the causal interpretation of sufficient combinations that are not necessary as well as for deriving necessary conditions that are not sufficient, if they are empirically consistent, empirically important, and substantively important. The SI approach prefers solution types that risk including redundant conditions over solutions that risk excluded relevant conditions. The RFapproach focuses on what can be inferred from the data and strictly prioritizes redundancyfree models, which only include the conditions for which the data provides evidence that they are relevant. Hereby, solutions interpreted in the RF-approach might include incomplete conjuncts, sufficient combinations from which causally relevant conditions have been eliminated.

The question arises to what extent these different ways of thinking about solution types in QCA have been explicitly linked to an underlying theory of causation that would provide them with a methodological basis.

\section{Theories of causation and qualitative comparative analysis}

The state of the art does not allow us to conclusively attribute all QCA approaches to a given theory of causation. However, we review the extant literature in view of how the issues discussed above have been linked to theories of causation, identify open questions and possible limitations. We do not approach this question by delving on different paradigms of ontology (questions about the existence of a real and objective world) (Della Porta and Keating 2008). QCA as an analytic approach has not been associated with a specific ontology or epistemology so far (Berg-Schlosser et al. 2009).

Instead, we delve into the research-practical implications of theories about causal thinking and causal inferences in the social sciences (Brady 2008). In line with Rohlfing and Zuber (2019), we argue that approaches to explanation in QCA — and related preferences for solution types-need to coherently and explicitly state what makes causal claims true, establish so-called truth conditions allowing one to separate causal from non-causal 


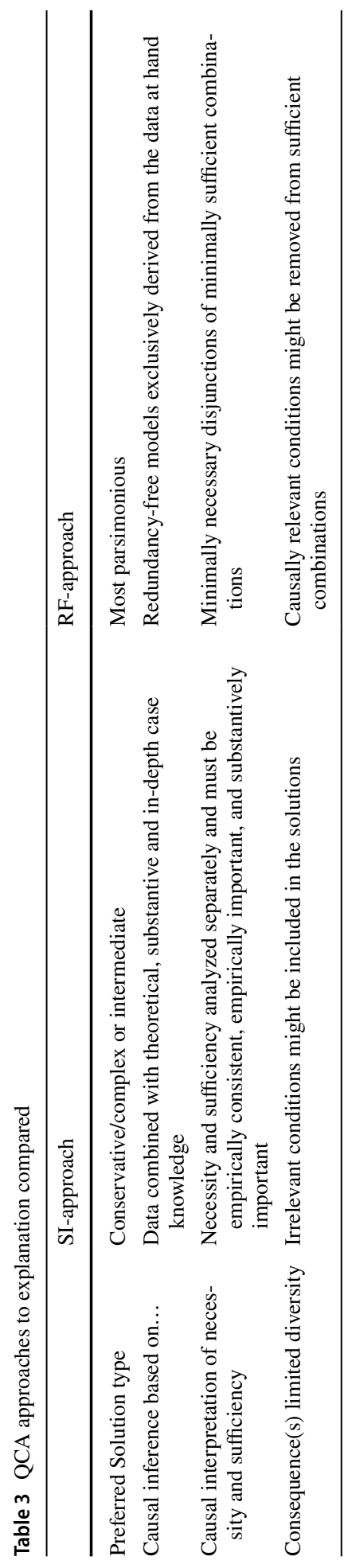


relationships, and align causal inferences and methodological recommendations with theories of causation. A general overview of different theories of causation has already been provided elsewhere (cf. e.g. Baumgartner 2020; Johnson et al. 2019; Rohlfing and Zuber 2019); we discuss their relevance for different QCA approaches. This section first discusses two dimensions on which theories of causation differ, after which four theories of causation that have been linked to QCA are discussed: regularity, type-level counterfactual, token-level counterfactual, and mechanistic theories of causation. ${ }^{3}$

\subsection{Variation between theories of causation}

\subsubsection{Type-level or token-level causation}

First, two kinds of causation can be distinguished (Baumgartner 2020). General or type causation (1) refers to relationships between classes of events or types of cases. Singular or token causation (2) refers to relationships between singular, instantiated events or cases (Rohlfing and Zuber 2019). 'Studying is causally relevant for passing an exam' is an example of a type-level causal claim. 'Peter passed the exam because he studied hard' is an example of a token-level causal claim. General and singular causation are clearly not independent (Baumgartner 2020). If studying is causally relevant for passing, there will be specific cases in which studying was (part of) the cause of passing. Vice versa, if Peter passed a specific exam because he studied, there might be a type-level causal structure that features studying as general cause of passing. Researchers can situate truth conditions at the token level and still additionally study type-level relationships, and vice versa; the important question is whether we need to demonstrate a causal relationship at the token or the type level (Rohlfing and Zuber 2019). Relationships at the other level, then, are merely a generalization or an instantiation of that causal relationship.

QCA's "technical moment", which builds on truth tables and logical minimization, seems to operate at the type level of cross-case comparison; however, it is still possible to allocate truth conditions for causal (as opposed to descriptive) inferences on an additional basis (Rubinson et al. 2019). Originally, QCA belongs to the case-oriented tradition (Rihoux 2013, 237) which strongly emphasizes the need for inferences at the token level. Ragin (1987/2014, 120) argues that "the results of Boolean analysis do not take the place of interpretive analysis; the task of applying the results to cases remains once a solution has been obtained". Likewise, Rihoux and De Meur $(2009,65)$ argue that the crucial last step of QCA, the interpretation of the results, requires a "return to the cases". Schneider $(2018,253)$ argues that "no QCA solution [...] should count as causal in the absence of within-case evidence of the presence of a causal mechanism". Hereby, the truth conditions of causal claims are located at the token-level. In contrast, condition-oriented QCA derives inferences at the type level. Examples and types of cases are used to illustrate the "instantiation of a type-level causal inference" (Rohlfing and Zuber 2019, 26). As Table 1 illustrates, most of the contributions of this special issue situate the truth condition for causal claims with QCA at the type level. However, Mahoney and Acosta (2021) as well as Alamos-Concha et al. (2021) also see a need to identify a spatio-temporal connection or a causal process at the within-case level.

\footnotetext{
${ }^{3}$ Probabilistic approaches to causation are incompatible with CCMs (Thiem et al. 2016) and therefore not discussed.
} 


\subsubsection{Difference-making or production}

A second difference between theories of causation is that they can either apply a criterion of difference-making or a criterion of production for establishing causality (Paul et al. 2013). Difference-making means that "an event counts as a cause when its presence or absence makes a difference to the presence or absence of the effect" (Rohlfing and Zuber $2019,6)$. The technical moment of QCA is geared towards identifying difference-makers for the outcome. Conversely, a production view on causation takes an event as a cause when it is connected to the outcome through a process or chain of events (Rohlfing and Zuber 2019). While identifying this is not possible through technical QCA, a case-oriented approach that requires the verification of causal mechanisms underlying QCA solutions might be compatible with a production view on causation.

\subsection{Regularity theories of causation}

When conceiving causes as regularities, causal relationships must meet three criteria (Mahoney and Acosta 2021): the cause temporally precedes the effect; cause and effect are proximate in time and space (spatio-temporal contiguity); and crucially, the effect regularly follows the cause (Psillos 2009). QCA has been explicitly associated with regularity theories of causation (RTC) (Mello 2021). There are indeed convincing arguments in favor of underpinning CCMs with a RTC (Baumgartner 2015). First of all, regularity theories are geared towards finding type-level causal relations and invoke a criterion of differencemaking for establishing causal relations. This is coherent with the analytical techniques used in QCA and CNA, which operate at the level of cross-case comparison and identify difference-makers for the outcome. In addition, QCA is geared towards finding necessary and sufficient relations, which fits well with a regularity understanding of causation (Mello 2021). Lastly, as shown in the work of Baumgartner (2015), interpreting parsimonious solutions as regularity causes is formally correct and coherent.

The RF-approach to QCA and CNA are firmly rooted in a RTC that builds on modern regularity theories that go back to John Mackie's INUS-theory (Mackie 1965, 1974) and contend that causally relevant conditions are indispensable parts of sufficient combinations, which are themselves non-redudant parts of necessary disjunctions, implying that causes are "boolean difference-makers" for their effects (Baumgartner and Falk 2019; Graßhoff and May 2001). Identifying such boolean difference-makers requires finding minimally necessary disjunctions of minimally sufficient combinations. Thus, the RF-approach contends that only a parsimonious solution with a high coverage can be interpreted causally, because only this solution corresponds to a minimally necessary disjunction of minimally sufficient combinations (Baumgartner and Ambühl 2018).

In their contribution to this special issue, Mahoney and Acosta (2021) suggest that both the RF-approach and SI-approach can be rooted in RTC. Mahoney and Acosta (2021) argue that QCA solutions can be causally interpreted if they are complete and redundancyfree. However, as they acknowledge, arriving at complete and redundancy-free solutions is extremely difficult in the social sciences. Baumgartner (2021) provides empirical support for the latter claim. By introducing the concept of a minimally robustly sufficient condition, Baumgartner (2021) provides an exact definition of a solution that is both complete and redundancy-free. The simulations presented in this contribution show that QCA solutions are unlikely to present minimally robustly sufficient combinations if the data feature high fragmentation or noise. 
More generally, Mahoney and Acosta (2021) develop a regularity theory of causation for the social sciences, focusing on the three criteria mention at the outset of this section: temporal order, spatiotemporal connection and constant conjunction. QCA scholars have mainly been concerned with the last criterion. However, Mahoney and Acosta (2021) argue that QCA scholars would benefit from also examining the requirement of spatiotemporal contact by combining QCA with within-case analysis. This allows to associate regularity theories with QCA without freeing the method from its traditional case-orientation. Another major contribution of Mahoney and Acosta (2021) is to show that regularity theories are not necessarily incompatible with considering counterfactual cases to make causal inferences (Mello 2021).

\subsubsection{Limitations}

Considering QCA as a method that is geared towards identifying causal relations in terms of type-level regularities has a number of downsides. First, under a RTC, QCA only guarantees correct causal inferences under the assumption of configurational homogeneity, which is anything but guaranteed in applied research. Usually, there are potentially relevant conditions that vary between the cases and are not included in the analysis. If researchers want to include all these conditions in their analysis, their solutions risk becoming too complex and/or too ambiguous to allow for meaningful interpretation. By requiring very high consistency and coverage levels and the assumption of configurational homogeneity, the RF approach to QCA has troubles in accounting for the shortcomings of "noisy", that is, often inconsistent and incomplete empirical data (Schneider 2018).

Lastly, QCA solutions are frequently incomplete because of limited empirical diversity of reality. According to Mahoney and Acosta (2021), the SI- approach deals with the practical difficulties for finding complete and redundancy-free solutions by combining QCA with case-studies. The contribution by Baumgartner (2021), in turn, makes clear that the RF-approach aims to identify causally relevant conditions rather than finding "complete" solutions or "robustly" sufficient conditions.

\subsection{Type-level counterfactual and interventionist theories}

Generally speaking, "a counterfactual is a statement, typically in the subjunctive mood, in which a false or 'counter to fact' premise is followed by some assertion about what would have happened if the premise were true" (Brady 2008, 1). Woodward (2005), for instance, defines causes as follows: "A necessary and sufficient condition for $\mathrm{X}$ to be a direct cause of $\mathrm{Y}$ with respect to a variable set $\mathrm{V}$ is that there be a possible intervention on $\mathrm{X}$ that will change $\mathrm{Y}$ (or the probability distribution of $\mathrm{Y}$ ) when all other variables in $\mathrm{V}$ besides $\mathrm{X}$ and $\mathrm{Y}$ are held fixed at some value by interventions." (Woodward 2005, 55). Causal relationships carry a "hypothetical or counterfactual commitment" in Woodward's interventionist theory, given that "they describe what the response of $\mathrm{Y}$ would be if a certain sort of change in the value of X were to occur" (Woodward 2005, 40).

Arguably, the SI-approach to QCA strongly relies on type-level counterfactual assumptions. Truth table rows are interpreted as types of cases. In the Standard Analysis (Ragin 2008), counterfactuals (logical remainder rows) are compared to empirically observed truth table that are identical except regarding one condition, and the plausibility that this counterfactual row will produce the outcome is assessed. In a similar vein, Enhanced Standard Analysis is centered around the premise of excluding impossible interventions on $\mathrm{X}$-in 
the form of untenable counterfactuals-from the analysis (Schneider and Wagemann 2012; 2013). Thus, relying on (enhanced) intermediate solutions seems to imply that researchers treat truth table rows as type-level counterfactuals. In this vein, Alamos-Concha et al. (2021) refer to type-level counterfactuals at the moment of cross-case analysis with QCA.

In their contribution to this special issue, Mahoney and Owen (2021) argue that although QCA fits more naturally with a regularity theory of causality, the set-theoretic tools used in QCA for analyzing necessity and sufficiency can be imported in quantitative research, which is rooted in a counterfactual theory of causation. They explore how settheoretic methods from the qualitative research tradition can enrich multimethod quantitative research and develop a general set-theoretic framework for the study of necessity and sufficiency in quantitative research. They conclude that set-theoretic methods add value by calling attention to asymmetrical patterns in the data that otherwise go unnoticed and unanalyzed. According to them, elementary QCA methods can be used profitably by statistical researchers who are interested in studying the causal effects of treatments.

\subsubsection{Limitations}

A fully developed type-level counterfactual theory of causation that could underpin QCA is yet to be spelled out (Schneider and Rohlfing 2019, 5). Whether the SI-approach to QCA is fully consistent with type-level counterfactual theories, like Woodward's interventionism, has not yet been clarified by methodologists. Methods that are underpinned by type-level counterfactuals theories do not always rest on existing knowledge to construct counterfactuals as is done in the SI-approach. Quantitative methods based on the potential outcomes framework are underpinned by counterfactual theories, but assess treatment effects without using theoretical knowledge to construct type-level counterfactuals. In consequence, counterfactual theories would yet need to provide clear guidelines on how to incorporate existing knowledge in the analysis to separate causal from non-causal relations. Moreover, Mahoney and Acosta (2021) highlight that "with a population of cases, counterfactual theories of causality analyze probabilistic relationships among variables" using average treatment effects (ATE). It is not clear how a focus on ATE would be compatible with QCA (Thiem et al. 2016). Finally, it is anything but certain that a counterfactual theory of causation would not suffer the same limitations as regularity theories of causation. It seems particularly unlikely that a type-level counterfactual theory of causation would not require an assumption of causal homogeneity in some form or another (Woodward 2005, 60).

\subsection{Token-level counterfactuals}

Lewis $(1973,573)$ defines causation as counterfactual dependence at the token level: "If c and e are two actual events such that e would not have occurred without $\mathrm{c}$, then $\mathrm{c}$ is a cause of e". Counterfactual claims here refer to "non-actual scenarios" and enable researchers to construct a "possible world" similar to the empirical case which ideally only differs regarding x (Lewis 1973), or to use structural counterfactual theories to determine the best possible cases for empirical comparison (Rohlfing and Zuber 2019).

Token-level counterfactuals have been most explicitly linked to QCA in the unifying framework for set-theoretic multi-method research (SMMR) put forward by Rohlfing and Schneider (2018). QCA performs complementary functions in SMMR, which uses process tracing based on token-level counterfactuals to draw causal inferences about the completeness of INUS configurations, the causal relevance of conjuncts, and scope conditions. 
Methodological literature on QCA has also indirectly referred to token-level counterfactuals theories of causation. When introducing the intermediate solution, Ragin and Sonnett (2005, 184), for example, build on the literature on counterfactual thought experiments, which sometimes refers to Lewis' counterfactual theory of causation (cf. inter alia Fearon 1991). However, as argued in the contribution of Mahoney and Acosta (2021), scholars who embrace regularity theories of causation also use such counterfactual thought experiments to make causal inferences.

\subsubsection{Limitations}

A token-level counterfactual theory needs to be accommodated with the analytical procedures used in QCA, which involves type-level cross-case comparisons. The procedures for making counterfactual assumptions in the SI-approach, for example, require assigning outcome values to truth table rows, which are by definition at the type-level. When relying on token-level counterfactuals, the truth conditions for causal claims are located at the eventrather than type-level, and the inferences of QCA need to be verified through causal inferences via counterfactuals on the level of single cases to be causally interpretable (Rohlfing and Schneider 2018). Logical minimization can be used to identify conditions that are potential difference-makers and, thus, might be causally relevant. Subsequently, withincase analysis has to be used to test whether these potential difference-makers are indeed causally relevant. It would need to be clarified whether every condition of every causal path of a QCA-solution would need to be verified at the token level to be valid. If this were the case, one might question the added value of using QCA to draw causal inferences.

\subsection{Mechanistic theories}

Mechanistic theories of causation maintain that "two events are causally related when and only when they are connected by an intervening mechanism" (Glennan 2010, 317). A causal mechanism in this view is defined as "a theorized system that produced the outcome through the interaction of a series of parts that transmit causal forces from X to Y" (Beach 2018, 74). Mechanisms consist of entities that engage in activities that transmit causal forces from cause to outcome. Each part of the mechanism is necessary to give rise to the subsequent part. The parts should exhibit productive continuity, meaning that each of the parts logically leads to the next part, and there are not large logical holes in the causal story linking X and Y together (Beach and Pedersen 2016). Unlike the previous theories of causation, mechanistic theories do not consider causes as difference-makers but contend that causes produce their effects (Baumgartner 2020).

Few would argue that mechanistic causation can be directly uncovered with QCA, which applies analytical procedures to establish differences between types of cases. However, the presence of a causal mechanism can be found with process-tracing (PT) methods (Beach and Pedersen 2019; Pattyn et al. 2020). Under a mechanistic theory of causation, QCA cannot be used to establish causal relations, but it can be used instrumentally to "find potential causes, select appropriate cases for within-case analysis, and enable cautious generalizations about processes to small, bounded sets of cases" (Beach and Kaas 2020, 9). In their contribution to this special issue, Álamos-Concha et al. (2021) argue that the analytical goal of QCA, when combined with in-depth PT is to identify potential causally relevant conditions that lead to the occurrence of a phenomenon, making PT more robust. PT on its part enables one to disentangle how such conditions connect with the outcome. They 
Table 4 QCA approaches and potentially eligible theories of causation

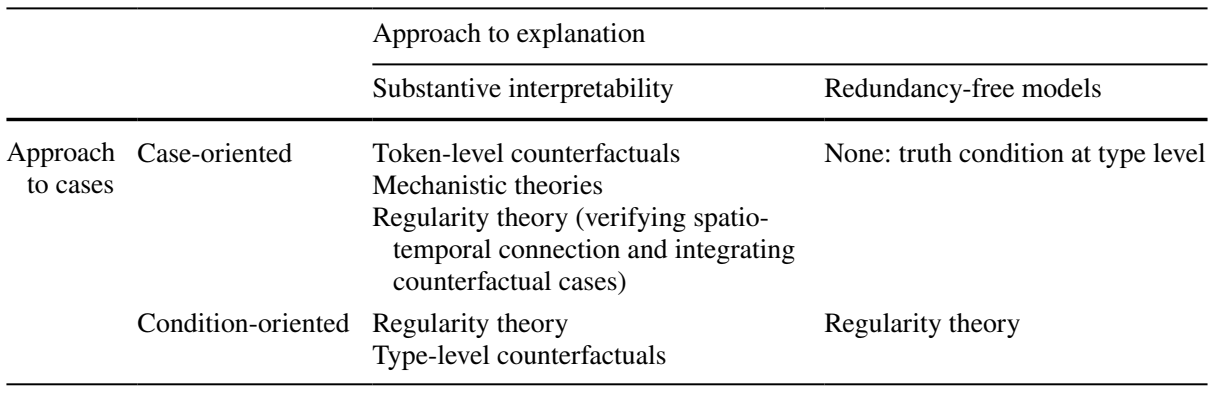

The table does not represent a conclusive attribution of causal theories to QCA approaches, but reflects the current proposals made in the field

discuss three challenges when following a mechanistic approach to causality in a multimethod research that combines QCA with in-depth process tracing: the risk of mechanistic heterogeneity, omitted conditions and challenges in generalization. Álamos-Concha et al. (2021) argue that the conservative solution is the most appropriate to mitigate these three challenges. It is most aligned with an understanding of causal mechanism as a system, and enables valid generalizations at the within-case level.

\subsubsection{Limitations}

As with token-level counterfactual theory of causation, a mechanistic understanding of causality needs to be accommodated with the type-level cross-case comparisons used in QCA. In this connection, the purpose of applying logical minimization is not entirely clear if QCA is used to complement process-tracing using a mechanistic approach. As argued in the article of Álamos-Concha et al. (2021), even the conservative solution can eliminate important contextual conditions. Moreover, the mechanistic variant of process tracing used to uncover such mechanisms differs from the counterfactual variant used in SMMR. Tracing mechanisms under a production view of causation requires within-case "mechanistic" evidence, rather than counterfactual assumptions on what could have happened in the absence of a condition (Beach 2018). In consequence, clear guidelines on how to deal with logical remainder rows cannot be straightforwardly deduced from mechanistic theories. A production view of causation generally cannot subscribe to the idea that the absence of a condition could be linked to producing an outcome (Rohlfing and Zuber 2019)—which is clearly one thing QCA researchers normally want to do.

\section{Conclusions and outlook}

In this special introduction we have mapped the debate on solution types with CCMs and related it to different approaches to QCA as well as potential links with underlying theories of causation. To summarize, our review shows that it is still not possible to conclusively attribute the SI-approach to QCA to a specific theory of causation. There are limitations in linking QCA with all four potentially eligible theories of causation. However, we have argued that it is important for QCA scholars to more explicitly state the truth conditions 
for their causal claims. Table 4 summarizes our discussion of the current state of the art regarding the potential attribution of QCA approaches to underlying theories of causation. Such an attribution has not conclusively been done yet for the SI approach. However, the table highlights some combinations that can be ruled out when considering that case-oriented QCA situates the truth condition for causal claims at least partly at the token level -in addition to the technical moment at the cross-case level-, while this is not required for condition-oriented QCA.

The special issue contributions have moved forward the state of the art in important ways: by proposing a regularity theory of causation for all QCA approaches (Mahoney and Acosta 2021); by enhancing our knowledge about different results of CNA and QCA (Swiatczak 2021); through a concrete proposal of a search target that is intended to be compatible with the philosophy of the SI-approach to QCA (Baumgartner 2021); by identifying an appropriate solution type for QCA-in-depth PT multimethod designs applying a mechanistic understanding of causality at the token level (Alamos-Concha et al. 2021); and by outlining how set-theoretic methodology can fruitfully inform quantitative research on the counterfactual tradition (Mahoney and Owen 2021).

Future research should address various open questions that prevail in this discussion. While the RF-approach to QCA and CNA draws on a regularity theory of causation, the causal basis of the SI approach is still a matter of scholarly exploration. Scholars are still to agree on an explicit formal definition of the search target under an SI-approach to QCA, and whether or not necessary but not sufficient conditions should be causally interpreted (Braumoeller and Goertz 2000; Goertz 2003; Mahoney and Acosta 2021). Arguably none of the theories of causation that we discussed would consider a necessary but not sufficient condition to be a cause. Future research should clarify how the RTC proposed by Mahoney and Acosta (2021), which situates the truth condition of causal claims at the type level but integrates token-level inferences and counterfactual cases, relates to the unifying framework for SMMR relying on token-level counterfactuals proposed by Rohlfing and Schneider (2018). Fifth, QCA methodologists should explore how the assumptions made on logical remainders under an SI-approach to QCA can be reconciled with counterfactual, regularity, or mechanistic theories of causation.

The RF-approach, in turn, recognizes that applying QCA to limited diverse data can result in incomplete models, but still has to clarify how the conclusions drawn from these incomplete models can be generalized. It is also not yet clear how researchers that want to follow the RF-approach can apply QCA to noisy and/or fragmented data (Schneider 2018). While the SI-approach suggests to build on theoretical and in-depth case knowledge (Mahoney and Owen 2021), the RF-approach has yet to provide practical guidelines on how QCA can be used to draw causal inferences from less-than-perfect data. This special issue is intended not only to clarify and structure the parameters of this debate, but also to contribute to the advancement of developing criteria for valid causal inferences in the field of CCMs.

\section{References}

Álamos-Concha, P., Pattyn, V., Rihoux, B., Schalembier, B., Beach, D., Cambré, B.: Conservative solutions for progress: on solution types when combining QCA with in-depth process-tracing. Qual. Quant. (2021)

Baumgartner, M.: Inferring causal complexity. Sociol. Methods Res. 38(1), 71-101 (2009)

Baumgartner, M.: Detecting causal chains in small-n data. Field Methods 25(1), 3-24 (2013) 
Baumgartner, M.: Parsimony and causality. Qual. Quant. 49(2), 839-856 (2015)

Baumgartner, M.: Causation. In: Berg-Schlosser, D., Badie, B., Morlino, L. (eds.) The SAGE Handbook of Political Science, pp. 305-321. Sage, London (2020)

Baumgartner, M.: Qualitative Comparative Analysis and robust sufficiency. Qual. Quan. (2021)

Baumgartner, M., Ambühl, M.: Causal modeling with multi-value and fuzzy-set coincidence analysis. Polit. Sci. Res. Methods 1-17 (2018)

Baumgartner, M., Falk, C.: Boolean difference-making: a modern regularity theory of causation. Br. J. Philos. Sci. (2019). https://doi.org/10.1093/bjps/axz047

Baumgartner, M., Thiem, A.: Often trusted but never (properly) tested: evaluating qualitative comparative analysis. Sociol. Methods Res. 49(2), 279-311 (2020)

Beach, D.: Achieving methodological alignment when combining QCA and process tracing in practice. Sociol. Methods Res. 47(1), 64-99 (2018)

Beach, D., Kaas, J.G.: The great divides: incommensurability, the impossibility of mixed-methodology, and what to do about it. Int Stud Rev (2020)

Beach, D., Pedersen, R.B.: Causal case study methods: foundations and guidelines for comparing, matching, and tracing. University of Michigan Press, Ann Arbor (2016)

Beach, D., Pedersen, R.B.: Process-tracing Methods: Foundations and Guidelines. University of Michigan Press, Ann Arbor (2019)

Berg-Schlosser, D., De Meur, G., Ragin, C., Rihoux, B.: Qualitative comparative analysis (QCA) as an approach. In: Rihoux, B., Ragin, C. (eds.) Configurational Comparative Methods, pp. 1-19. Sage, London (2009)

Brady, H.E.: Causation and explanation in social science. In: Goodin, R. (ed.) The Oxford Handbook of Political Science. Oxford University Press, Oxford (2008)

Braumoeller, B.F., Goertz, G.: The methodology of necessary conditions. Am. J. Polit. Sci. 844-858 (2000)

Della Porta, D., Keating, M.: How many approaches in the social sciences? An epistemological introduction. In: Della Porta, D., Keating, M. (eds.) Approaches and Methodologies in the Social Sciences: A Pluralist Perspective, pp. 19-39. Cambridge University Press, Cambridge (2008)

Duşa, A.: QCA with R: A Comprehensive Resource. Springer, Cham (2018)

Dușa, A.: Critical tension: sufficiency and parsimony in QCA. Sociol. Methods Re. (2019). https://doi. org/10.1177/0049124119882456

Fearon, J.D.: Counterfactuals and hypothesis testing in political science. World Politics 43(2), 169-195 (1991)

Fiss, P.C.: Building better causal theories: A fuzzy set approach to typologies in organization research. Acad. Manag. J. 54(2), 393-420 (2011)

Glennan, S.: Mechanisms. In: Beebee, H., Hitchcock, C., Peter, M. (eds.) The Oxford Handbook of Causation. Oxford University Press, Oxford (2010)

Goertz, G.: Cause, correlation, and necessary conditions. In Necessary Conditions: Theory, Methodology, and Applications, 47-64 (2003)

Graßhoff, G., May, M.: Causal regularities. In: Spohn, W., Ledwig, M., Esfeld, M. (eds.) Current Issues in Causation. pp. 85-114. Mentis, Hardehausen (2001)

Greckhamer, T., Misangyi, V.F., Fiss, P.C.: The two QCAs: From a small-N to a large-N set theoretic approach. In: Fiss, P.C., Cambré, B., Marx, A. (eds.) Configurational theory and methods in organizational research, pp. 49-75. Emerald Publishing, Bingley (2013)

Haesebrouck, T.: Relevant, irrelevant or ambiguous? Towards a correct interpretation of QCA's solution types. Accepted at Sociol. Methods Res. (2021) (forthcoming)

Johnson, R.B., Russo, F., Schoonenboom, J.: Causation in mixed methods research: The meeting of philosophy, science, and practice. J. Mixed Methods Res. 13(2), 143-162 (2019)

Lewis, D.: Causation. J. Philos. 70(17), 556-567 (1973)

Mackie, J.: Causes and conditions. Am. Philos. Qurterly. 2(4), 245-264 (1965)

Mackie, J.: The Cement of the Universe: A Study of Causation. Oxford University Press, Oxford (1974)

Mahoney, J., Acosta, L.: A Regularity Theory of Causality for the Social Sciences. Quality \& Quantity (2021)

Mahoney, J., Owen, A.: Importing Set-Theoretic Tools into Quantitative Research: The Case of Necessary and Sufficient Conditions. Quality \& Quantity (2021)

Mello, P.A.: Qualitative Comparative Analysis: An Introduction to Research Design and Application. Georgetown University Press, Washington DC (2021)

Oana, I.-E., Schneider, C.Q., Thomann, E.: Qualitative Comparative Analysis (QCA) Using R: A Gentle Introduction. Cambridge University Press, Cambridge (2021) 
Pattyn, V., Álamos-Concha, P., Cambré, B., Rihoux, B., Schalembier, B.: Policy effectiveness through configurational and mechanistic lenses: lessons for concept development. J. Compar. Policy Analy. Res. Pract. 1-18 (2020). doi:https://doi.org/10.1080/13876988.2020.1773263

Paul, L.A., Hall, N., Hall, E.J.: Causation: A User's Guide. Oxford University Press (2013)

Psillos, S.: Regularity theories. In: Beebee, H., Hitchcock, C., Peter, M. (eds.) The Oxford Handbook of Causation. Oxford (2009)

Radaelli, C.M., Wagemann, C.: What did I leave out? Omitted variables in regression and qualitative comparative analysis. Eur. Polit. Sci. 18(2), 275-290 (2019). https://doi.org/10.1057/ s41304-017-0142-7

Ragin, C.: The Comparative Method: Moving Beyond Qualitative and Quantitative Methods. University of California Press, Berkeley (1987/2014)

Ragin, C.: Redesigning Social Inquiry: Fuzzy Sets and Beyond. University of Chicago Press, Chicago (2008)

Ragin, C., Rihoux, B.: Configurational Comparative Methods: Qualitative Comparative Analysis (QCA) and Related Techniques. Sage, London (2009)

Ragin, C., Sonnett, J.: Between complexity and parsimony: Limited diversity, counterfactual cases, and comparative analysis. In: Kropp, S., Minkenberg, M. (eds.) Vergleichen in der Politikwissenschaft, pp. 180-197. Springer, Cham (2005)

Rihoux, B.: Qualitative comparative analysis (QCA), anno 2013: reframing the comparative method's seminal statements. Swiss Polit. Sci. Rev. 19(2), 233-245 (2013)

Rihoux, B., De Meur, G.: Crisp-set qualitative comparative analysis (csQCA). In: Ragin, C., Rihoux, B. (eds.) Configurational Comparative Methods: Qualitative Comparative Analysis (QCA) and Related Techniques, vol. 51, pp. 33-68. (2009)

Rihoux, B., Lobe, B.: The case-orientedness of qualitative comparative analysis (QCA): Glass halfempty or half-full. Teorija in Praksa 52(6), 1039-1245 (2015)

Rohlfing, I., Schneider, C.Q.: A unifying framework for causal analysis in set-theoretic multimethod research. Sociol. Methods Res. 47(1), 37-63 (2018)

Rohlfing, I., Zuber, C.I.: Check your truth conditions! Clarifying the relationship between theories of causation and social science methods for causal inference. Sociol. Methods Res. (2019). https://doi. org/10.1177/0049124119826156

Rubinson, C., Gerrits, L., Rutten, R., Greckhamer, T.: Avoiding Common Errors in QCA: A Short Guide for New Practitioners.9, 397-418 (2019)

Rutten, R.: Applying and assessing large-N QCA: causality and robustness from a critical realist perspective. Sociol. Methods Res. 0049124120914955 (2020)

Schneider, C.Q.: Realists and Idealists in QCA. Polit. Anal. 26(2), 246-254 (2018)

Schneider, C.Q., Rohlfing, I.: Set-theoretic multimethod research: the role of test corridors and conjunctions for case selection. Swiss Polit. Sci. Rev. 25(3), 253-275 (2019)

Schneider, C.Q., Wagemann, C.: Set-Theoretic Methods for the Social Sciences: A Guide to Qualitative Comparative Analysis (QCA). Cambridge University Press, Cambridge (2012)

Schneider, C.Q., Wagemann, C.: Doing justice to logical remainders in QCA: moving beyond the standard analysis. Polit. Res. Quart. 211-220 (2013)

Swiatczak, M.: Different algorithms, different models. Qual. Quant. (2021)

Thiem, A.: Using qualitative comparative analysis for identifying causal chains in configurational data: a methodological commentary on Baumgartner and Epple (2014). Sociol. Methods Res. 44(4), 723736 (2015). https://doi.org/10.1177/0049124115589032

Thiem, A.: Beyond the facts: limited empirical diversity and causal inference in qualitative comparative analysis. Sociol. Methods Res. 0049124119882463 (2019). doi:https://doi.org/10.1177/00491 24119882463

Thiem, A., Baumgartner, M.: Back to square one: a reply to Munck, Paine, and Schneider. Comp. Pol. Stud. 49(6), 801-806 (2016). https://doi.org/10.1177/0010414015626455

Thiem, A., Baumgartner, M., Bol, D.: Still lost in translation! A correction of three misunderstandings between configurational comparativists and regressional analysts. Comp. Pol. Stud. 49(6), 742-774 (2016)

Thiem, A., Mkrtchyan, L., Haesebrouck, T., Sanchez, D.: Algorithmic bias in social research: a metaanalysis. Plos ONE 15(6), e0233625 (2020)

Thomann, E.: Qualitative comparative analysis (QCA) as an approach for comparative policy analysis. In: Peters, G., Falk, M., Fontaine, G. (eds.) Handbook of Research Methods and Applications in Comparative Policy Analysis. Edward Elgar Publishing, Northampton (2020) 
Thomann, E., Ege, J.: Qualitative comparative analysis (QCA) in public administration. In: Peters, G., Thynne, I. (eds.) Oxford encyclopedia of public administration. Oxford University Press, Oxford (2020)

Thomann, E., Maggetti, M.: Designing research with qualitative comparative analysis (QCA) approaches, challenges, and tools. Sociol. Methods Res. 49(2), 356-386 (2020)

Woodward, J.: Making things happen: a theory of causal explanation. Oxford University Press, Oxford (2005)

Publisher's Note Springer Nature remains neutral with regard to jurisdictional claims in published maps and institutional affiliations. 